\title{
Microscale torsion test to investigate initial yielding phenomenon of a single crystal copper
}

\author{
T. Yokoyama, ${ }^{* 1}$ N. Shishido, $*^{1}, *^{2}$ K. Koiwa ${ }^{* 1, * 2}$, S. Kamiya, ${ }^{* 1, * 2}$ H. Sato, ${ }^{* 1, * 2}$ M. Omiya, ${ }^{* 2}, *^{3}$ \\ M. Nishida, ${ }^{* 1}{ }^{12}$ T. Suzuki, ${ }^{* 4}$ T. Nakamura, ${ }^{4}$ T. Nokuo, ${ }^{2}{ }^{2}{ }^{5}$ and T. Suzuki ${ }^{2}, *^{5}$ \\ *11 Nagoya Institute of Technology, Gokiso-cho, Showa-ku, Nagoya, Japan \\ $*^{2}$ Japan Science and Technology Agency, Chiyoda-ku, Tokyo, Japan \\ *3 Keio University, 3-14-1 Hiyoshi, Kohoku-ku, Yokohama, Japan \\ ${ }^{*}$ Fujitsu Laboratories Ltd., 10-1 Morinosato-Wakamiya Atugi, Japan \\ *5 JEOL Ltd, 3-1-2 Musashino, Akishima, Japan
}

\begin{abstract}
Mechanical property of copper is very important for the reliable design of semiconductor devices, because the interface between a copper line and the dielectric barrier layer is the week point in the device. In general, the line width of copper line is in the range from tens nanometer to micrometer, and the plastic property of a crystalline metal with small dimensions is highly sensitive to its dimension [1]. In addition, it appears that the loading condition significantly affects the plastic behavior of a micron scale metal. C Motz et al found the follow stress of almost $1 \mathrm{GPa}$ (i.e. nearly equal to theoretical strength of copper crystal) at the micro-bending test [2]. Considering these reports, in order to comprehend deformation behavior of the copper embedded in semiconductor device, we need to investigate mechanical response of the copper with small dimensions under heterogeneous stress distribution. In this paper, we twisted micron copper specimens with different dimensions, and investigated the size effect of its plasticity, especially focused on the initial yielding.
\end{abstract}

Fig. 1(a) shows the specimen subjected to the torsion test with the small dimensions from submicron to microns. A single crystal plate of copper (purity $99.9999 \%$ and $\{110\}$ surface orientation) was prepared by cutting out of the ingot grown by Czochralski method. The copper plate was milled by focused $\mathrm{Ga}^{+}$ion beam to shape the specimen. The arc-shape on the $\{110\}$ plane was formed by FIB milling from the top, and the bottom of the arc was removed from the lateral face. Finally, FIB stripped off the surface layer of $200 \mathrm{~nm}$ thickness from the specimen with $10 \mathrm{pA}$ and $30 \mathrm{kV}$ of $\mathrm{Ga}^{+}$beam in order to reduce the damage originated by $\mathrm{Ga}^{+}$ bombardment. The dimension of the fabricated specimen was represented by the specimen side length $a$ depicted on Fig. 1(b). Three different types of specimens were successfully fabricated with $a=0.3 \mu \mathrm{m}, 1.0 \mu \mathrm{m}$, and $1.5 \mu \mathrm{m}$. The stress distribution was shown in Fig. 1(c). Microscale torsion test was conducted inside the scanning electron microscope (JIB4600F, JEOL Ltd., Japan). Fig.2 shows the secondary electron image of the torsion test observed with the acceleration voltage of $15 \mathrm{kV}$. The specimen was pushed on the end of the arc shape by the nanoindenter (PI87, Hysitron Inc., USA), which can measure load and displacement. The applied displacement of the nanoindenter was controlled with a constant rate of $10 \mathrm{~nm} / \mathrm{s}$. Load-displacement curve of the specimen with $a=1.0 \mu \mathrm{m}$ was shown in Fig. 3. The linear part of the obtained curve corresponds to the fully elastic response of the specimen. The proportional limit of the linear part can be defined as the initial yielding point, circled in Fig. 3 .

Maximum resolved shear stress on the slip system of the copper crystal at the initial yield point was calculated as the initial yield stress of the corresponding specimens. The obtained yield stress was plotted in Fig. 4. The cross section of the specimen twisted by the maximum torque is (001) plane and the slip plane with maximum resolved shear stress is (-111) as shown in Fig. 4 in yellow and in red, respectively. The yield stress is considerably higher than the bulk [3], and we confirmed the size effect on it. And, the obtained result from torsion test also showed the apparently-similar tendency to that of compression test [4]. However, compression is deformed uniformly, and torsion is deformed starting from the surface of specimen. This difference of morphology is expected to affect the size effect of initial yielding behavior. 
References

[1] M D Uchic, et al., Science 305 (2004), p. 986-989.

[2] C Motz, T Schoberl, R Pippan., Acta Maerialia 53 (2005), p. 4269-4279.

[3] M F Horstemeyer, et al., Journal of Engineering Material and Technology 124 (2002), p. 322-328.

[4] D Kiener, A M Minor, Acta Materialia 59 (2011), p. 1328-1337

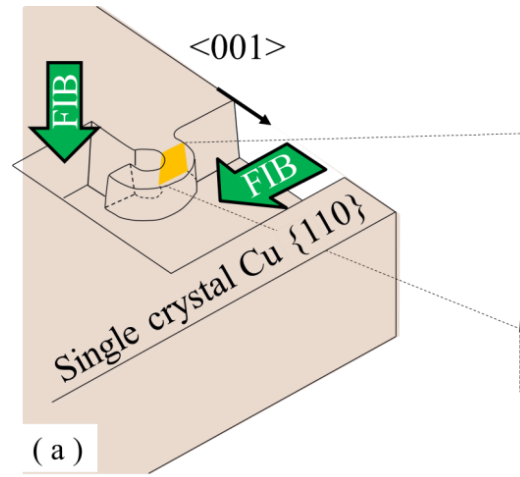

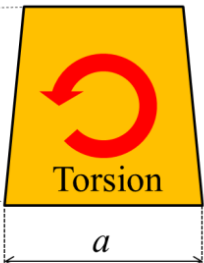

( b )

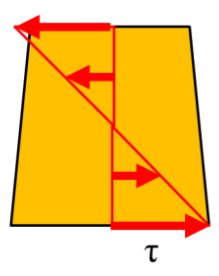

(c)

Figure 1. The specimen subjected to the torsion test; (a) Specimen fabrication by FIB milling, out of a single crystal copper. (b) The specimen side length $a$ represents the specimen dimensions. (c) The stress distribution of cross section has maximum torque.
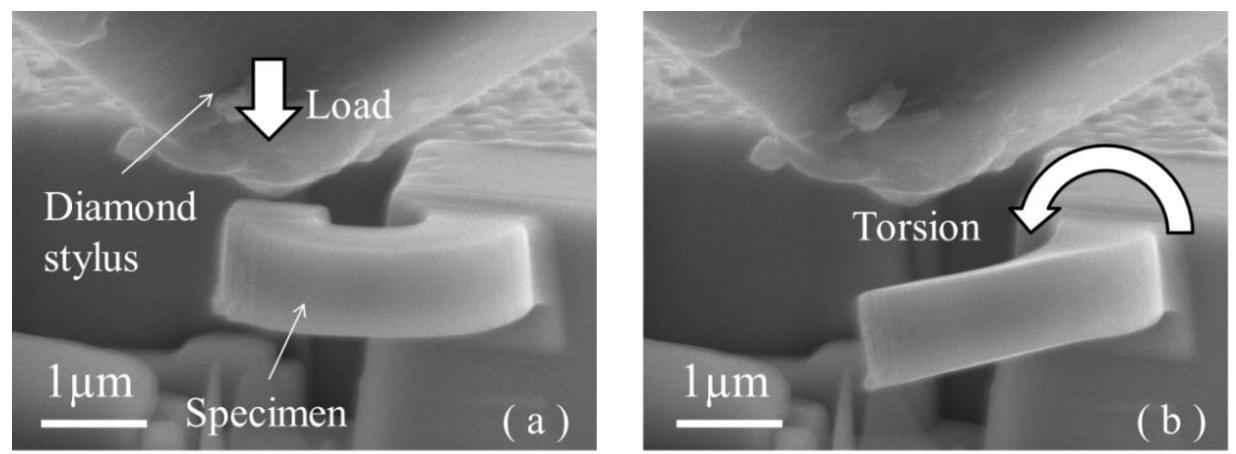

Figure 2. SEM image of $1 \mu \mathrm{m}$ specimen during torsion test; (a) Before twisting (b) After twisting.

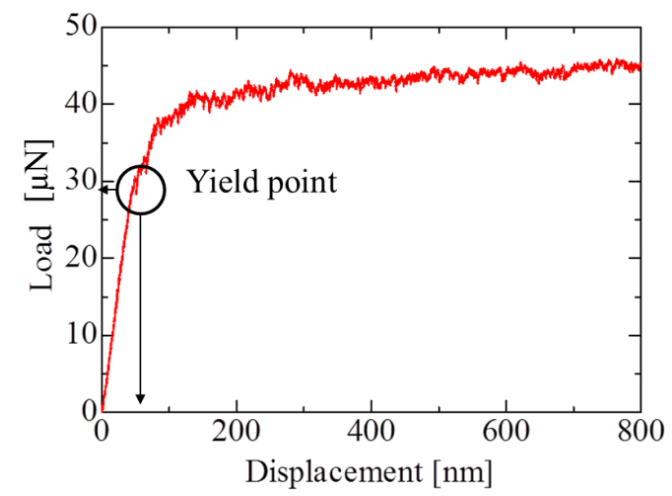

Figure 3. The load-displacement curve of the specimen with $a=1.0 \mu \mathrm{m}$

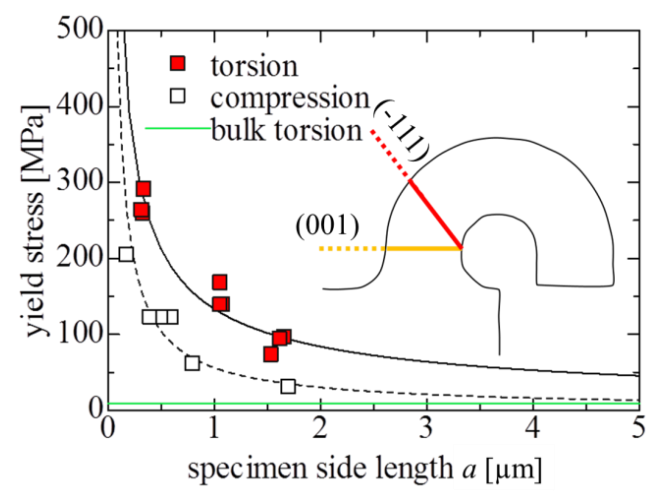

Figure 4. Initial yield stress among different specimen side length 